\title{
Seroepidemiology of Human Polyomaviruses
}

\author{
Jaime M. Kean ${ }^{1}$, Suchitra Rao ${ }^{2}$, Michael Wang ${ }^{2}$, Robert L. Garcea ${ }^{1,2,3 *}$
}

1 Department of Microbiology, University of Colorado School of Medicine, Denver, Colorado, United States of America, 2 Department of Pediatrics, University of Colorado School of Medicine, Denver, Colorado, United States of America, 3 Department of Molecular, Cellular, and Developmental Biology, University of Colorado, Boulder, Colorado, United States of America

\begin{abstract}
In addition to the previously characterized viruses BK and JC, three new human polyomaviruses (Pys) have been recently identified: KIV, WUV, and Merkel Cell Py (MCV). Using an ELISA employing recombinant VP1 capsid proteins, we have determined the seroprevalence of KIV, WUV, and MCV, along with BKV and JCV, and the monkey viruses SV40 and LPV. Soluble VP1 proteins were used to assess crossreactivity between viruses. We found the seroprevalence $(+/-1 \%)$ in healthy adult blood donors (1501) was SV40 (9\%), BKV (82\%), JCV (39\%), LPV (15\%), KIV (55\%), WUV (69\%), MCV strain 350 (25\%), and MCV strain $339(42 \%)$. Competition assays detected no sero-crossreactivity between the VP1 proteins of LPV or MCV or between WUV and KIV. There was considerable sero-crossreactivity between SV40 and BKV, and to a lesser extent, between SV40 and JCV VP1 proteins. After correcting for crossreactivity, the SV40 seroprevalence was $\sim 2 \%$. The seroprevalence in children under 21 years of age $(n=721)$ for all Pys was similar to that of the adult population, suggesting that primary exposure to these viruses likely occurs in childhood.
\end{abstract}

Citation: Kean JM, Rao S, Wang M, Garcea RL (2009) Seroepidemiology of Human Polyomaviruses. PLoS Pathog 5(3): e1000363. doi:10.1371/journal.ppat.1000363 Editor: Walter J. Atwood, Brown University, United States of America

Received December 29, 2008; Accepted March 2, 2009; Published March 27, 2009

Copyright: (C) 2009 Kean et al. This is an open-access article distributed under the terms of the Creative Commons Attribution License, which permits unrestricted use, distribution, and reproduction in any medium, provided the original author and source are credited.

Funding: Support was provided by NIH/NIAID grant T32 AI52066 (J.M.K.), NIH/NCI CA37667 (R.L.G.), American Cancer Society/University of Colorado Pilot Grant ACS IRG 57-001-47 (M.W.), St. Baldrick's Foundation (M.W.), Prestige Miracles Foundation (M.W.), and the Cancer League of Colorado, Inc. (M.W.). The funders had no role in study design, data collection and analysis, decision to publish, or preparation of the manuscript.

Competing Interests: The authors have declared that no competing interests exist.

*E-mail: Robert.Garcea@colorado.edu

\section{Introduction}

Polyomaviruses are small, non-enveloped dsDNA viruses that occupy replicative niches in a variety of vertebrates, and have been extensively studied as oncogenic agents in experimental systems. Five human polyomaviruses have now been identified: BKV [1], JCV [2], KIV [3], WUV [4], and most recently, Merkel Cell Polyomavirus (MCV) [5]. BKV and JCV were discovered in 1971 $[1,2]$ and are apparently ubiquitous as determined by serology studies, infecting over $80 \%$ of some populations by adulthood. Primary infections with BKV and JCV are still not well characterized. Both BKV and JCV persist for life, and their tissue sanctuaries may include mononuclear blood cells (BKV, JCV) and cells of the proximal renal tubule (BKV). Reactivation of these viruses in immunocompromised individuals, results in hemorrhagic cystitis, nephropathy (BKV) and progressive multifocal leukoencephalopathy (JCV).

KIV and WUV were isolated from respiratory specimens by PGR methods, indicating a potential for both disease and transmission via the respiratory route. They have been detected in populations from 4 continents, suggesting a global distribution [6-14]. PCR evidence suggests a low prevalence for both KIV and WUV genomes in respiratory samples from individuals [15]. Neither KIV nor WUV DNA has been detected in blood or urine, however, KIV has been detected in fecal specimens [3].

MCV was recently discovered in a Merkel cell carcinoma, integrated into the host cell genome in a manner suggesting a possible relationship to oncogenesis [5]. Interestingly, MCV has a close sequence relationship to the primate Lymphotropic Polyomavirus (LPV). LPV was first isolated in 1979 from an African Green Monkey B-lymphoblastoid cell line [16]. Gellular tropism for LPV includes continuous lines of B lymphoblasts of both human and monkey origin [17-19]. Original serologic evidence suggested that an LPV-like virus infection may occur in humans and more recent assays using specific $\mathrm{VP} 1$ reagents have suggested that $15-25 \%$ of humans are seropositive for LPV VPl-reactive antibodies [20], indicating that exposure to LPV or a virus antigenically related to LPV occurs in the human population. The sequence similarity between LPV and MCV raises the possibility that the seroepidemiology of LPV may be coincident with that of MCV. Another primate virus, SV40, also has been detected in human tissues, but its prevalence and relationship to human disease is controversial.

Little is known about the primary illness associated with infection or potential disease associations of the newly discovered human polyomaviruses. Serologic studies indicate that exposure to BKV and JCV initially occurs during childhood, however, it is unknown when exposure occurs for LPV, KIV, WUV and MCV. In order to study exposure to these viruses in humans, we used recombinant polyomavirus VP1 capsid proteins expressed in E. coli in an ELISA assay similar to that described previously for HPV serotype analysis $[21,22]$. Our serological results provide data on the prevalence and age-related timeline for infection with the recently discovered polyomaviruses, KIV, WUV, and MCV, as well as for those previously identified: SV40, LPV, BKV, and JCV.

\section{Results}

\section{Seroprevalence in an adult population}

Sera from 1501 adults over the age of 21 were tested for VP1reactive antibodies using recombinant $\mathrm{VPl}$ capsomeres from SV40, BKV, JCV, LPV, MCV, KIV, and WUV. Seroprevalence 


\section{Author Summary}

Polyomaviruses occupy a replicative niche in animals from birds to humans. Two human polyomaviruses, BKV and JCV, were discovered in 1971 and within the last two years, three new polyomaviruses have been found in humans: KI (KIV), WU (WUV), and Merkel Cell (MCV) polyomavirus. MCV was identified in Merkel Cell carcinomas, a rare skin cancer. To date, it has not been determined what percentage of the human population is exposed to KIV, WUV, and MCV, and when initial exposure to these viruses occurs. We determined that initial exposure to KIV, WUV, and MCV occurs in childhood, similar to that for the known human polyomaviruses BKV and JCV, and that their prevalence is high. We also found evidence that a monkey virus, Lymphotropic Polyomavirus (LPV), likely has a serologically related human counterpart. Another monkey polyomavirus, SV40, was found at $\sim 2 \%$ prevalence, a level that does not support its role in human disease.

values for KIV and WUV were 55\% and 69\%, respectively. Although seroprevalence values for both KIV and WUV were high in our population, infection with these viruses was not always coincident. We detected differential seroreactivity to 2 different isolates of MCV: 350 (25\%) and 339 (46\%). Before assessment of crossreactivity, we detected a seroprevalence of $9 \%$ for SV40 and $82 \%$ for BKV. These values are consistent with previous reports $[23,24]$. Thirty-nine percent of the population had JCV VP1reactive antibodies, and $15 \%$ of the population had antibodies against LPV VP1. The LPV seroprevalence was not coincident with the MCV seroprevalence for either isolate, and the respective VP1 proteins did not compete for seroreactivity, as described below. Our data indicate that there may be an age-related waning of BKV VP1 specific antibodies, however, our data do not suggest an age-related waning for any of the other 6 polyomaviruses tested (Figure 1). We did not detect a difference in seroprevalence with respect to gender for any of the 7 polyomaviruses tested (Table 1).

\section{Age-specific seroprevalence in a pediatric population}

The pediatric population consisted of 721 study subjects under the age of 21. As shown in Figure 1, seroprevalence values for these individuals were SV40 (9\%), BKV(73\%), JCV (21\%), LPV (14\%), MCV isolate 350 (23\%) and MCV isolate 339 (34\%), KIV $(56 \%)$ and WUV (54\%). Antibodies to all the VP1 antigens tested were detected in children between 1 and 3 years of age in our study population (Table 1). These data indicate that exposure to all 7 polyomaviruses may occur early in childhood.

\section{Antibody crossreactivity determined using competition with soluble VP1 proteins}

Our assay allows testing for possible crossreactivity between VP1 proteins by preincubation of sera with soluble VP1 proteins prior to the specific ELISA antigen. No serologic crossreactivity was detected between the VP1 proteins of LPV and either isolate of MCV or between the VPl proteins of KIV and WUV (Figure 2). Interestingly, there were 164 sera in our population that were seroreactive to the VP1 protein of MCV isolate 350 , but not to 339. Also, 560 samples were seroreactive to isolate 339 , but not to isolate 350. Antibody crossreactivity was observed between the VP1 proteins of SV40 and BKV. Therefore, competition assays with soluble BKV and JCV VP1 capsomeres were performed for all SV40 positive samples to determine the extent of SV40 seroreactivity due to crossreactivity with these other viruses. Two hundred samples were found to have SV40 VPl-reactive antibodies, 5 of these samples were not found to have seroreactivity to the $\mathrm{VPl}$ proteins of either BKV or JCV, while 195 samples exhibited coincident seroprevalences with SV40 and BKV and/or JCV (Figure 3). Of the 195 coincident samples, 83 (43\%) samples were competed using VP1 pentamers from both BKV and JCV, while $62(32 \%)$ samples were competed using only BKV VPl pentamers, and 7 (3\%) samples were competed using only JCV VP1 pentamers (Figure 3). The ELISA reactivity of 43 $(22 \%)$ of the 195 coincident samples were not competed with VP1 capsomeres of either BKV and/or JCV. Therefore, a total of 48 samples ( $2 \%$ of the study population) exhibited SV40 "specific" antibodies (Figure 3). Of these 48 samples, 16 (33\%) were from individuals less than 21 years of age, $20(42 \%)$ were obtained from individuals between the ages of 21 and 55 years, and $12(25 \%)$ were obtained from individuals over the age of 55 years (Table $\mathrm{S} 1$ ). It was not possible from these de-identified samples to determine if the latter group had received SV40-contaminated polio vaccine.

\section{Discussion}

We have determined the seroprevalence for three recently identified human polyomaviruses (KIV, WUV, and MCV) and confirmed the seroprevalence of two previously known human polyomaviruses (BKV and JCV) and two monkey polyomaviruses (SV40 and LPV) in a human population, using a VP1 capsomerebased ELISA. Our study evaluates both large adult $(\mathrm{n}=1501)$ and pediatric populations $(n=721)$ to determine the overall prevalence and age of first exposure to these viruses. Additionally, our assay evaluated potential serocrossreactivity occurring between these viruses.

Previous studies of human Py serology have used a variety of assays, including hemaglutination inhibition (HI) [25-28] and a virus-like particle (VLP) ELISA-based assay [20,29-31], which may not be directly comparable. The hemaglutination inhibition assay requires either intact virions or VLPs and only evaluates a subset of antibodies. HI may be less sensitive for determining BKV and JCV VP1 seroreactivity, as compared to enzyme immunoassays [32]. Moreover, not all polyomaviruses exhibit hemaglutination (e.g., SV40) and HA has not yet been assessed for KIV, WUV and MCV. A VLP-based ELISA may present conformational epitopes and increased specificity over HI. However, while the VLP-based assay may measure only a subset of antibodies, the capsomere assay has the advantage of measuring all VPl-reactive antibodies, and the use of a casein-glutathione conjugate sterically projects the capsomeres from the well surface, allowing their full exposure to the sera. Nonetheless, seroprevalence determinations are likely somewhat dependent on specific conditions of the assay.

Our observed seropositivity for both WUV (69\%) and KIV $(55 \%)$ was high, despite a low reported detection rate in respiratory tract isolates using PCR [7,12,13,33,34]. The PCR data likely represent active infection or ongoing co-infection, rather than overall exposure rates. From the age stratification data, it appears that primary infection with these viruses occurs during early childhood, with $35 \%$ positive between ages $1-3$ for WU and $32 \%$ positive for KIV. There was no cross-reactivity between WUV and KIV, which may have been expected given only $65 \%$ amino acid identity between their VP1 proteins [4].

We found differential seroreactivity to MCV isolates 350 (25\%) and $339(42 \%)$. However, these are not true viral isolates but rather PCR amplified sequences, since no infectious virus has yet been characterized for any of the new human polyomaviruses. The PCR amplifications may have detected defective genomes or variants with minor sequence mutations that occurred after viral integration (specific mutational events have been reported for the 
A

SV40 age-specific seroprevalence

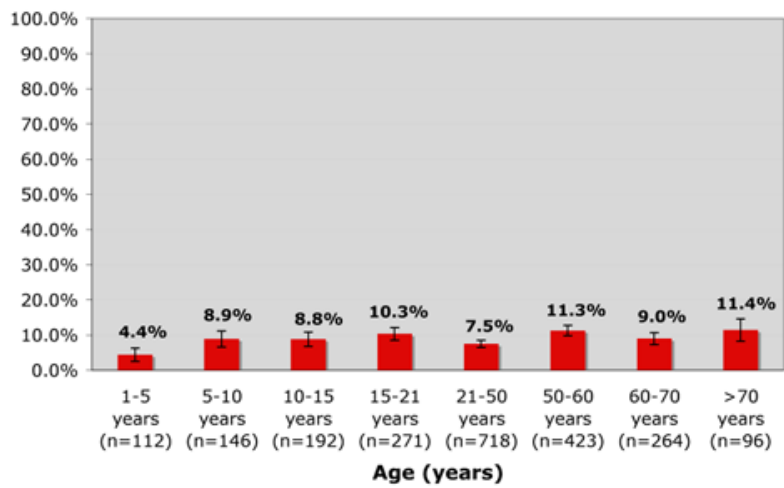

C

JCV age-specific seroprevalence

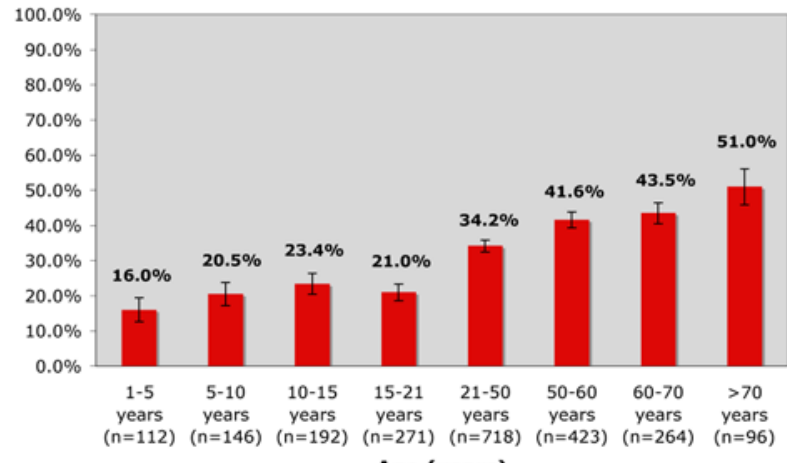

$E$

Age (years)

KIV age-specific seroprevalence

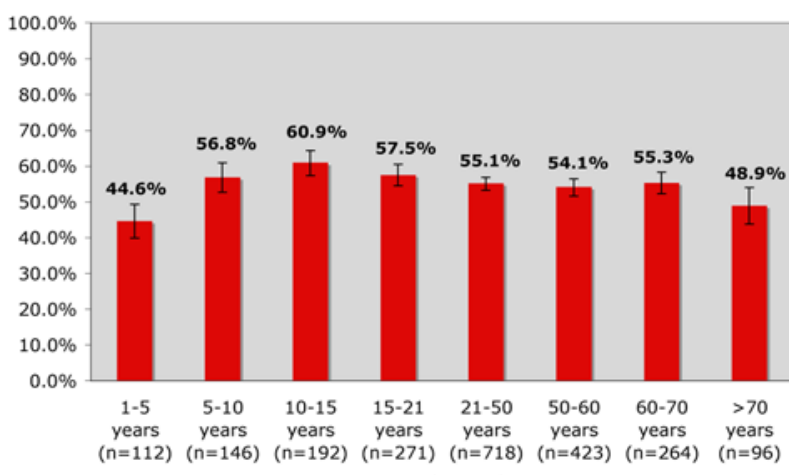

Age (years)

G

MCV isolate $\mathbf{3 5 0}$ age-specific seroprevalence

$\mathrm{H}$

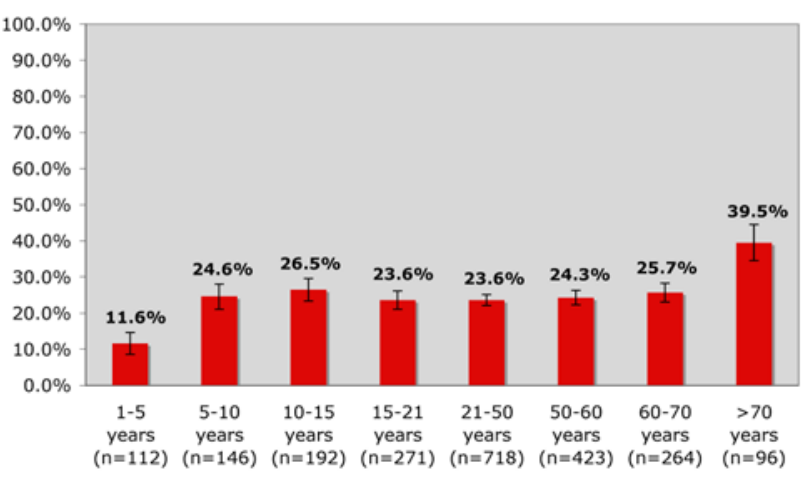

Age (years)

B

F

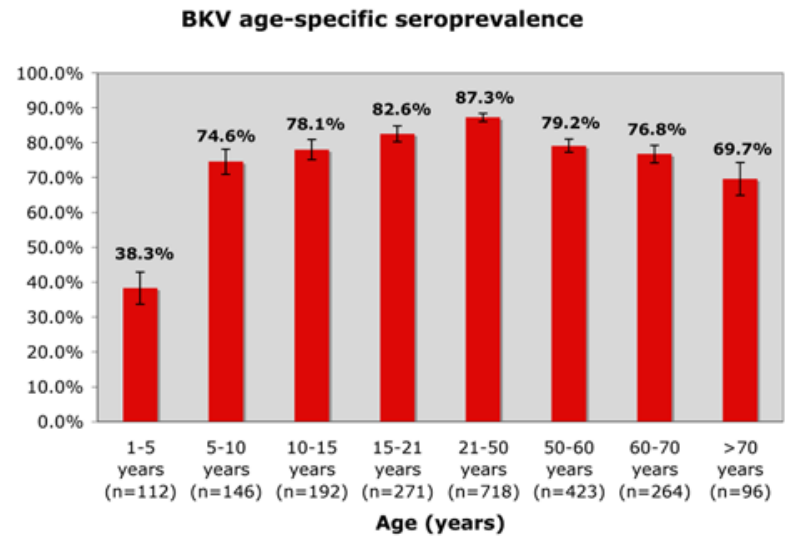

LPV age-specific seroprevalence

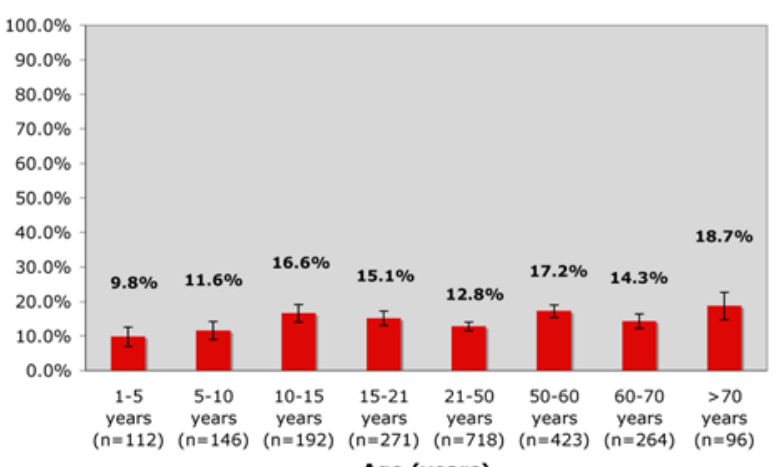

Age (years)

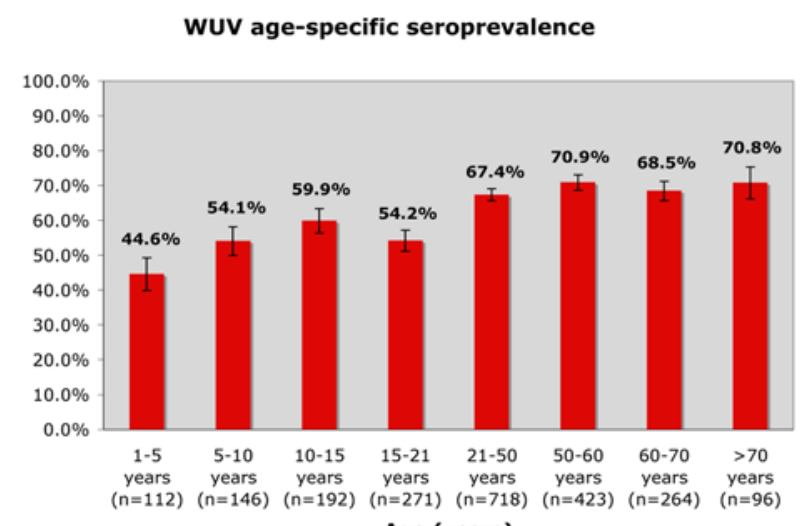

Age (years)

MCV isolate 339 age-specific seroprevalence

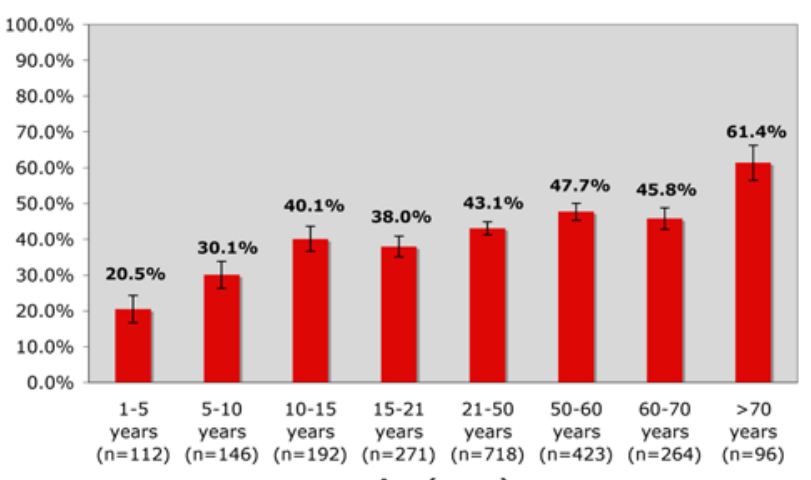

Age (years) 
Figure 1. Age-specific seroprevalence detected in a Denver, CO, USA study population $(n=2222)$ for 7 polyomaviruses known to infect humans. A) SV40 (seroreactivity prior to competition with BKV and JCV VP1 proteins; Figure 3, Table S1); B) BKV; C) JCV; D) LPV; E) KIV; F) WUV; G) MCV isolate 350; H) MCV isolate 339. Standard error bars are shown. doi:10.1371/journal.ppat.1000363.g001

Merkel large T-antigen protein [35], and VP1 mutations might be anticipated if they affected productive infection). The differential reactivity in our analysis may therefore have resulted from one PGR variant having a "less native" conformation of the recombinant VP1 protein used in the assays (although sufficiently native to generate VP1 pentamers by electron microscopy) or mutated in critical amino acids that affect a specific epitope. Interestingly, there were 164 sera in our population that were seroreactive to the VPl protein of MCV isolate 350 , but not to 339. Also, 560 samples were seroreactive to isolate 339 , but not to isolate 350 , suggesting that both isolates of MCV may circulate in the human population. It remains to be determined when authentic, infectious isolates are characterized whether there is actual strain variation in serology. However, as discussed below for JCV, strain differences likely will not substantially affect overall seroprevalence data.

If these are actually different strains of MCV, the differential prevalence may indicate differential geographic exposure frequencies to these MCV isolates [36,37]. Also, MCV isolate 339 may contain a sero-dominant MCV VP1 epitope not present in isolate 350. Five amino acid differences occur between the VP1 proteins of MCV 350 and 339. Based on alignment with the VP1 primary amino acid sequence of SV40 and the known structure of SV40 VP1, MCV 339 and 350 do not vary with respect to the surface VPl exposed variable loop regions thought to comprise the major antigenic determinants, however differences in amino acids occurring close to the surface may indirectly affect loop conformation (Figure S2). Specifically, H288 of MCV 350 is D288 in isolate 339 (Figure S2). Based on alignment with known SV40 VP1 structure, this amino acid difference occurs close to the surface near the HI variable loop. It remains to be determined whether the difference in seroprevalence between MCV isolates 350 and 339 is maintained in suspect disease populations. Our data also support previous studies suggesting that the human population has been exposed to an LPV-like virus, antigenically similar to the primate LPV VP1 [20,38], and LPV-like sequences have recently been detected in the white blood cells of immunocompromised individuals [39]. Although the recently discovered MCV exhibits a high degree of sequence similarity with LPV (Figure 4) our competition results indicate that MCV and LPV are antigenically distinct viruses.

While the seroprevalence of $\mathrm{BKV}$ in our study population is consistent with previous reports [23,24], the JCV seroprevalence $(39 \%)$ was somewhat lower $[30,31]$. There may be several reasons for this finding: 1) epidemiologic evidence suggests that JCV exposure may differ geographically [24], 2) JCV VP1- specific antibodies may not have as high affinity for the JCV VP1 proteins compared to BKV VPl-specific antibodies, resulting in an overall decreased sensitivity of the JCV assay, 3) although only the MAD$11 \mathrm{JCV}$ genotype has been found to be serologically distinct [40], our assay used only genotype $2 \mathrm{~B}$ and antibodies other than against genotype $2 \mathrm{~B}$ may bind that $\mathrm{VP1}$ protein with lower affinity, reducing the sensitivity of the assay.

BKV VP1 IgG antibodies previously have been observed to cross-react with SV40 VP1 [20]. Using recombinant VP1 proteins to compete potential seroreactivity between BKV and SV40, we found the seroprevalence of SV40 to be approximately $2 \%$ (Figure 3 and Table S1). In the population of individuals who were seropositive for SV40 and either BKV and/or JCV ( $\mathrm{n}=195)$,
SV40 seroreactivity could be competed with VP1 pentamers of both BKV and JCV in $43 \%$ of the coincident population. SV40 seroreactivity could be competed with BKV VPl capsomeres alone in $32 \%$ of coincident samples, while $3 \%$ of coincident samples could be competed only with JCV VP1 capsomeres. If there was no specific seroreactivity we did not assign seropositivity to SV40, since we assume that some antibodies must be specific to SV40 to indicate infection. Indeed, we identified 48 individuals with "specific" SV40 antibodies, supporting our assumption that the SV40 serological response is not restricted only to crossreactive antibodies with $\mathrm{BKV}$ or JCV. It is interesting to speculate that BKV infection might confer protection against SV40, an attribute that may have had a selective advantage at one time. Although we cannot explain the residual SV40 serology of $2 \%$, it is possible that a yet unidentified human polyomaviruses may account for this reactivity.

The external surface variable loop domains of the VP1 proteins may present the dominant epitopes of these viruses. For example, comparison of the loop regions of SV40 and BKV reveals a high degree of similarity. Specifically, the BC loops of SV40 and the BK strain of $\mathrm{BKV}$ share $53 \%$ identity, while the DE and HI loops exhibit $71 \%$ and $80 \%$ identity, respectively. Comparison of the loops of LPV and MCV, and those of KIV and WUV, reveals fewer similarities (Figure 4); LPV and MCV exhibit 21\% identity in the BC loop, and $26 \%$ and $20 \%$ in their respective DE and HI loops. KIV and WUV share $33 \%$ and $38 \%$ respective identity in the BC and DE loops, and only $20 \%$ identity in the HI loop. These differences may account for the lack of crossreactivity between these viruses.

Our pediatric population exhibits similar seroprevalence values compared to the adults, indicating that primary infection with polyomaviruses occurs in childhood (Figure 2). We found seropositivity to BKV, WUV, KIV, LPV, and MCV appears in early childhood whereas that for JCV occurs in pre-adolescence (Figure 1). Additionally, our data suggest there may be an agerelated waning of BKV VP1 specific antibodies, however, our data do not indicate an age-related waning for any of the other 6 polyomaviruses assayed (Figure 1). While the adult samples are likely representative of the demographics in the Denver area, and reflect healthy individuals within the guidelines for blood donation, the pediatric samples were obtained from inpatients and outpatients at The Children's Hospital Denver and although exclusion criteria were employed, some individuals may have had co-morbid or intercurrent illnesses.

Although animal models have suggested that polyomaviruses could be human tumor viruses [41-44], no substantive causeeffect relationships have yet been established. The debate over SV40 induction of human tumors remains controversial [45], and our data do not support a permissive human infection with SV40, such as seen with the other human polyomaviruses. If SV40 infects humans, it is very limited in scope. Furthermore, if a specific tumor type is presumed attributable to SV40 infection, serological validation now would be an essential factor in the analysis. The discovery of MCV integrated in a specific tumor [5] is a possible indication of the ability of these viruses to contribute to tumorigenesis, but perhaps only in a subset of cell types and only in immunosuppressed individuals. The identification of the human counterpart to LPV may also reveal a connection to human cancer. However, given the high seroprevalence of these viruses, 


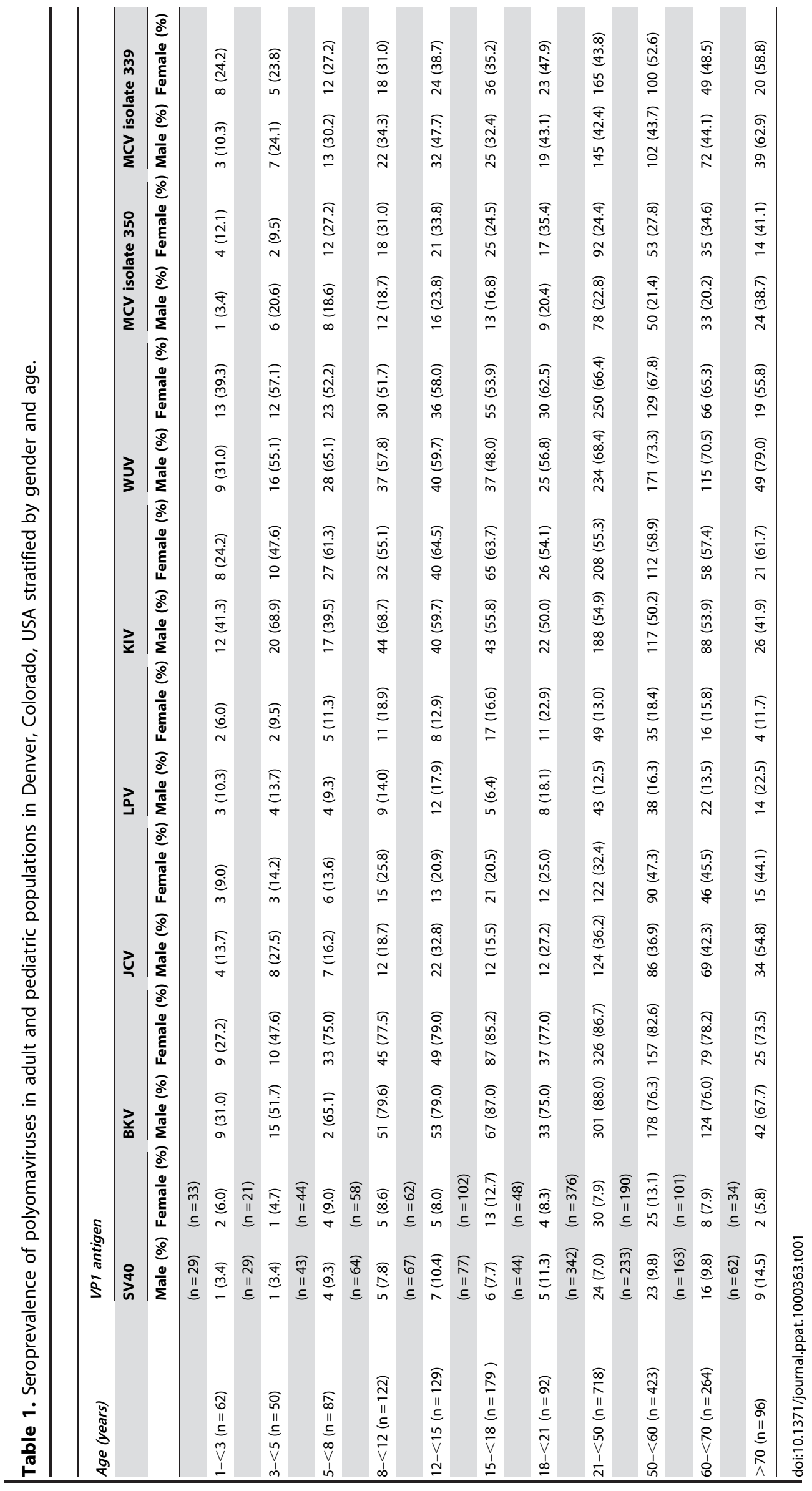


A

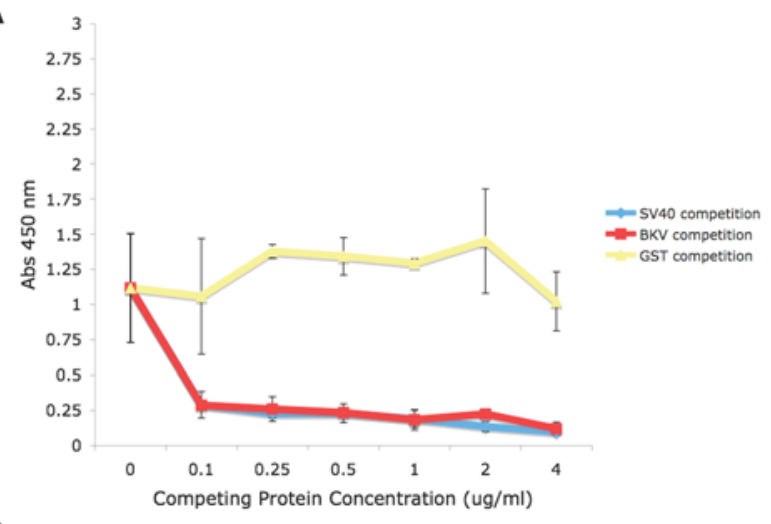

C

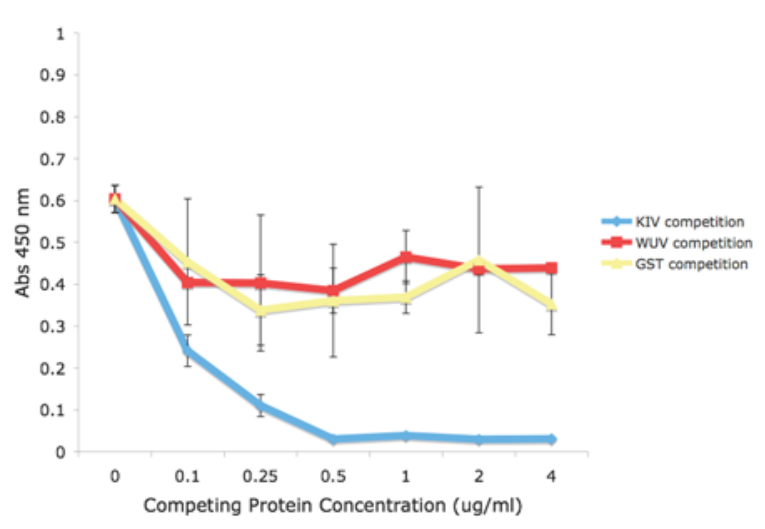

E

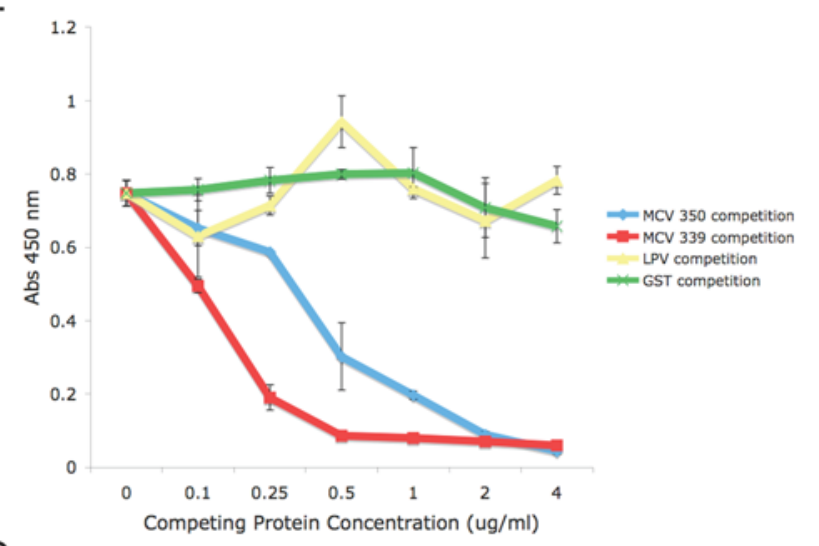

G

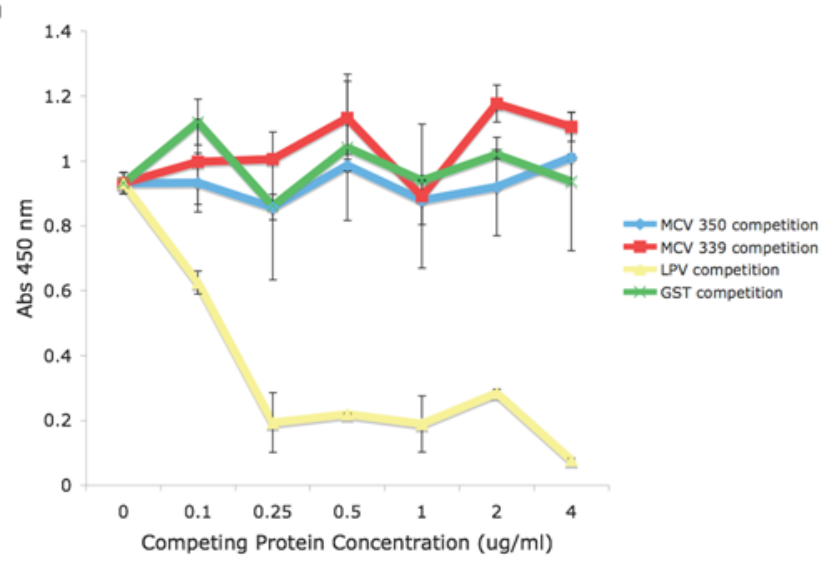

B

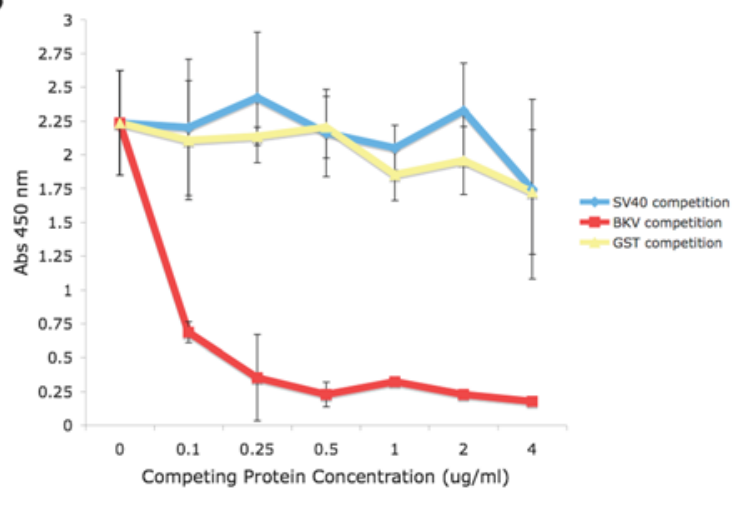

D
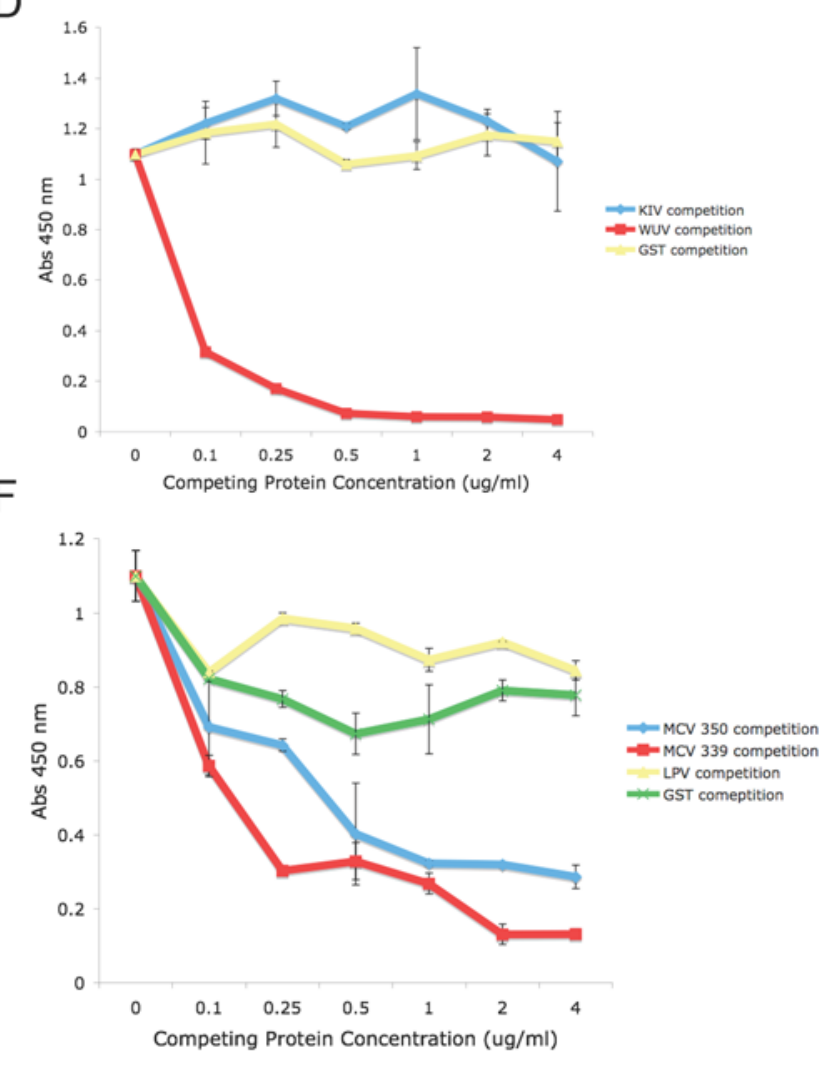

Figure 2. Seroreactivity to VP1 proteins after competition with soluble heterologous VP1 pentamers. A) SV40 seroreactivity competed with BKV; B) BKV seroreactivity competed with SV40; C) KIV seroreactivity competed with WUV; D) WUV seroreactivity competed with KIV; E) MCV isolate 350 seroreactivity competed with MCV isolate 339 and LPV; F) MCV isolate 339 seroreactivity competed with MCV isolate 350 and LPV; and G) LPV seroreactivity competed with MCV isolates 350 and 339. All capsid proteins were competed with themselves as well as with soluble GST as controls.

doi:10.1371/journal.ppat.1000363.g002 


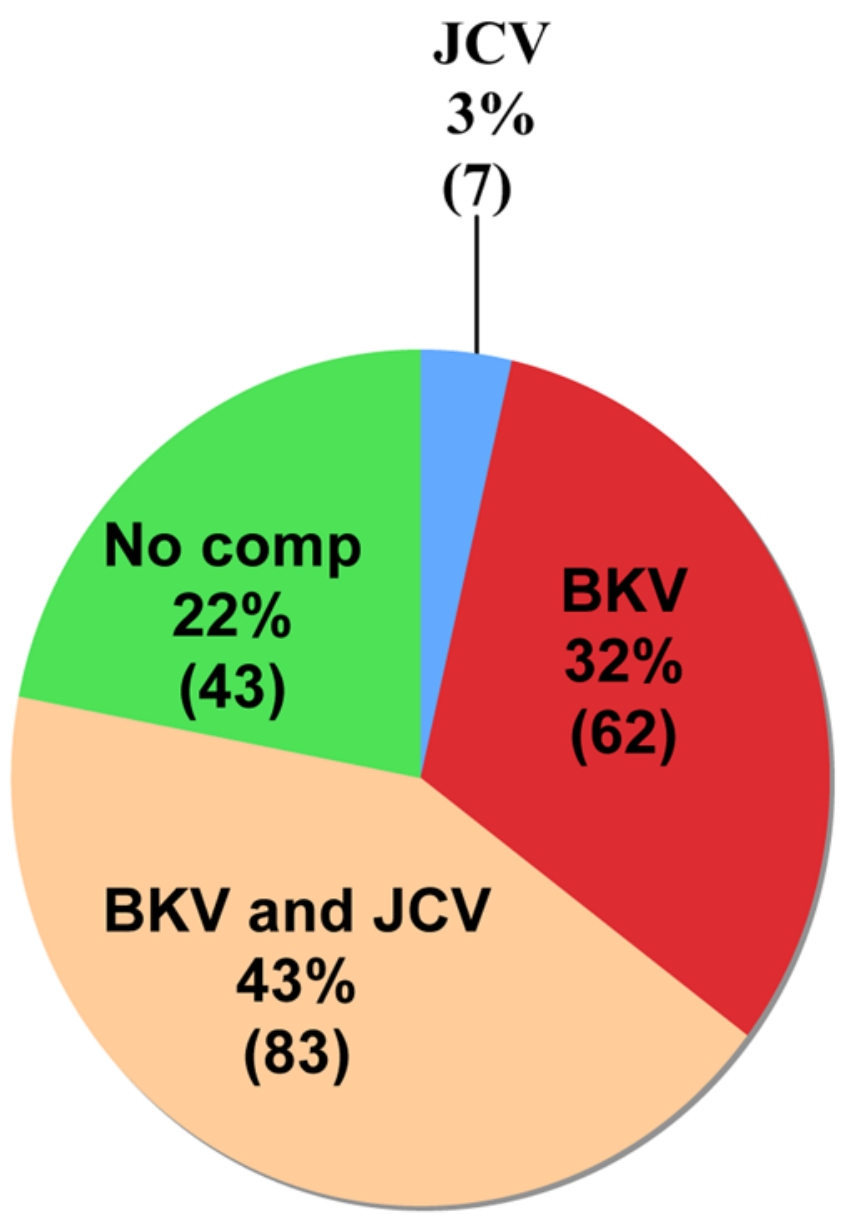

Figure 3. SV40 seroreactive samples competed with JCV and BKV VP1 proteins. 195 samples exhibiting initial SV40 seroreactivity (Figure 1A) were re-analyzed after pre-incubation with soluble BKV and/ or JCV VP1. The percentages indicate the number of samples for which SV40 seroreactivity was eliminated by the designated pre-incubation conditions. No competition was observed for 43/195 (23\%) of the samples.

doi:10.1371/journal.ppat.1000363.g003

serological support of etiologic connections to specific diseases may be problematic. Nonetheless, these ubiquitous viruses appear to be significant human pathogens in immunosuppressed populations.

\section{Materials and Methods}

\section{Ethics statement}

Plasma samples from healthy adult blood donors were obtained (May and June, 2007) from Bonfils Blood Center (Denver), and pediatric plasma samples were obtained from The Children's Hospital (Denver) using protocols approved by the Colorado Multiple Institutional Review Board. Consent from human participants was not obtained. Samples were de-identified and analyzed anonymously.

\section{Human plasma samples}

To ensure a healthy pediatric population for determination of baseline exposure, the pediatric population excluded patients who had received intravenous immunoglobulin in the preceding year and/or patients who had undergone blood, platelet, or plasma transfusions within two months of obtaining the sample. Children less than one year of age were excluded from the analysis because of possible confounding maternal antibodies.

\section{Expression plasmids for recombinant GST-VP1 fusion proteins}

pGEX4T3 plasmids (GE Healthcare) encoding the VP1 capsid proteins for SV40, LPV, BKV, JCV, KIV,WUV and MCV isolate 339 were obtained and subsequently used for protein production. The MCV isolate 350 vpl gene was synthesized according to the published sequence (Genbank accession number NG_010277) (Genscript) and subcloned into the BamHI and XhoI restriction sites of the pGEX4T3 expression vector (GE Healthcare). Subcloning was verified through DNA sequencing analysis of regions flanking the insert using the primers: $5^{\prime}$ GCATGGCCTTTGCAGGGC-3' and 5'-CGACACTGGCAGAGGCCC-3'.

\section{Expression and affinity purification of recombinant VP1 proteins}

VP1 proteins from SV40, BKV (BK strain), JCV (genotype 2B), LPV, KIV, WUV and MCV were expressed in E. coli and purified using affinity chromatography. Briefly, $400 \mathrm{ml}$ cultures of E. coli containing the pGEX VPl expression plasmids were grown at $37^{\circ} \mathrm{C}$ until $\mathrm{OD}_{600}=4.0$. Cultures were cooled to room temperature and induced with $1.25 \mathrm{mM}$ IPTG. Cells were then grown at room temperature to an $\mathrm{OD}_{600}$ of 8.0. Cells were lysed using a French Press. A clarified lysate was chromatographed using a GSTrap FF affinity column (GE Healthcare) to bind recombinant GST-VP1 capsomeres. Columns were washed with 3 column volumes of Superdex buffer (40 mM HEPES, pH 7.4; $200 \mathrm{mM}$ $\mathrm{NaCl}$; 5\% glycerol; $1 \mathrm{mM}$ EDTA, $5 \mathrm{mM}$ DTT, pH 7.2), and GST-VP1 capsomeres were eluted from the column with 10 column volumes of Superdex buffer supplemented with $10 \mathrm{mM}$ reduced glutathione. Protein fractions were combined, buffer exchanged, aliquoted, and stored at $-80^{\circ} \mathrm{C}$ for use in capture ELISA assays.

Soluble VP1 proteins used in competition assays were purified as described above. After buffer exchange, GST-VP1 capsomeres were incubated with glutathione sepharose beads (GE Healthcare) for $1 \mathrm{hr}$ at $4^{\circ} \mathrm{C}$. GST tags were cleaved with $50 \mathrm{U}$ of thrombin in $10 \mathrm{ml}$ lysis buffer (40 mM HEPES, pH 7.4; $200 \mathrm{mM} \mathrm{NaCl} ; 5 \%$ glycerol; $1 \mathrm{mM}$ EDTA), supplemented with $10 \mathrm{mM}$ DTT. Cleaved VP1 pentamers were collected, concentrated, aliquoted, and stored at $-80^{\circ} \mathrm{C}$ for use in competition assays.

\section{GST-VP1 capture ELISA}

N-terminal GST-tagged pentameric VP1 capsomeres were captured on polysorp 96-well plates (Nunc) using a caseinglutathione conjugate [22]. Plasma samples were diluted 1:50 in block buffer $(5 \%(\mathrm{w} / \mathrm{v})$ evaporated milk powder; $0.05 \%(\mathrm{v} / \mathrm{v})$ Tween 20 in phosphate buffered saline, $\mathrm{pH}$ 7.4) and incubated with the immobilized GST-VP1 antigen. IgG antibodies were detected using an HRP-labeled secondary antibody, and tetramethylbenzidine with hydrogen peroxide as a substrate. Positive control antibodies were: anti-LPV VP1 monoclonal antibody for LPV VP1 antigen and human sample 637 for both MCV VP1 antigens. We provided BKV VP1 pentamers to Bio-Synthesis Inc, for production of an anti-BKV polyclonal antibody. At a concentration of 1:50,000, the BKV polyclonal rabbit sera reacted with SV40, BKV, JCV, KIV, and WUV VP1 antigens. Negative controls included capture of GST alone and subsequent incubation with control antibodies for each antigen. ELISA assays for 90 samples were repeated for all 8 antigens tested. 
A

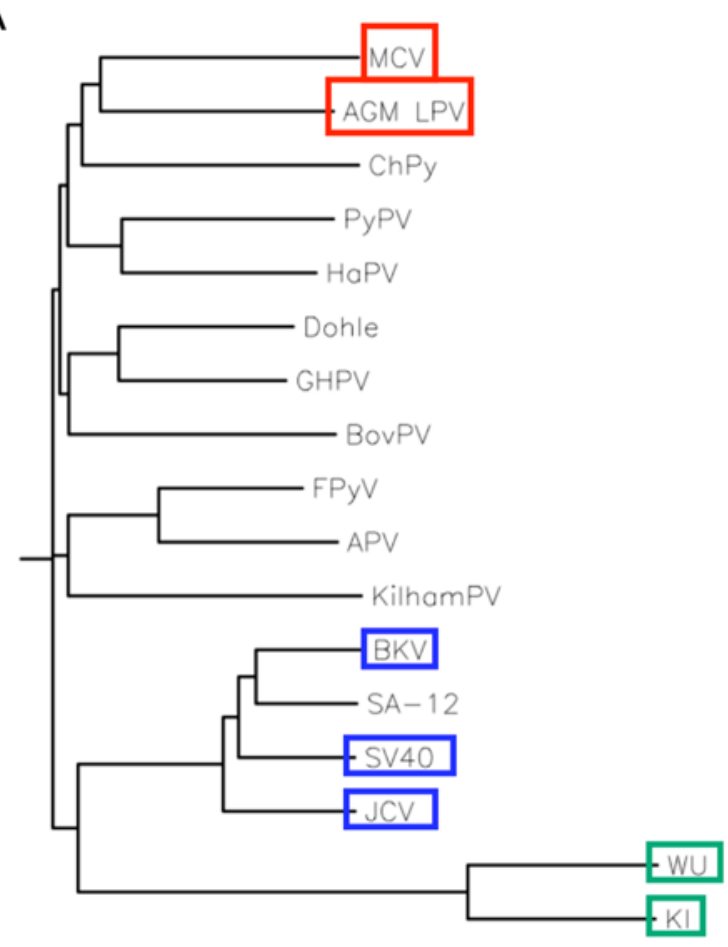

C $\begin{array}{ll}\text { BKV strain BK BC loop } & \text { GF SLKLSAENDFSSDS P } \\ \text { SV40 BC loop } & \text { GLSKSLAAEKQFTDDS P }\end{array}$

BKV strain BK DE loop L HA GS Q KV HEH G G G K P I SV40 DE loop LHSGTQKTHENGAGKPI

BKV strain BK HI loop N S S GT SV40 HI loop NTSGT

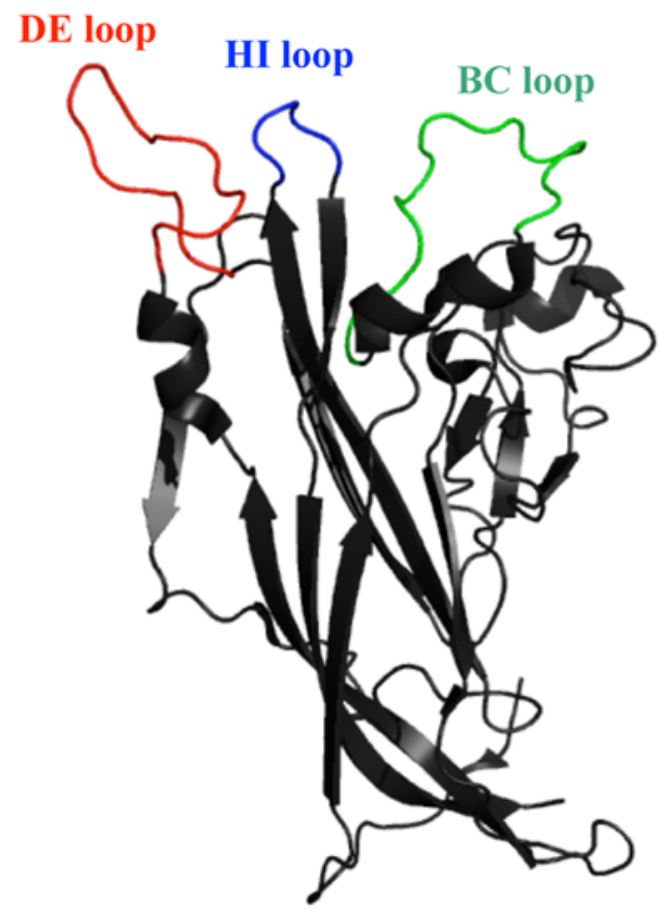

B

LPV BC loop LYGYSNS INTAFSKASDTP WYTYTYDLQPK-GSSPDQP

LPV DE loop MCV DE loop

LHQ-GGKY I YGSSSGCVPV VHYWDMKRVHDYGAG-I PV

LPV HI loop NYSET MCV HI loop $\quad$ KTS $\mathbf{S K}$

WUV BC loop - - - - - HYWS I S P LRTAEAAN-----VTP

KI BC loop GREPTPHYWSISSAIHDKESGSSIKVEETP

WUV DE loop - AGILTTGGVGGI

KI DE loop ALGN-TNGVVHGI

WUV HI loop GVNKN

KI HI loop GTANS

Figure 4. Genetic variability of VP1 proteins among polyomaviruses. A) phylogenetic analysis of 17 known polyomaviruses [45]: B) Crystal structure of VP1 monomer derived from pdb id 3BWQ. PyMOL [46] was used to illustrate the surface variable loop regions C) amino acid primary sequence alignments of VP1 variable loop regions for SV40 and BKV, LPV and MCV, and KIV and WUV.

doi:10.1371/journal.ppat.1000363.g004 
Reproducibility was assessed based on agreement of seropositivity for each sample run on different days. Kappa statistics indicated a high degree of agreement between samples: SV40 (0.8515), LPV (0.8218), BKV (0.8668), JCV (0.8062), WUV (0.9076), KIV (0.8851), MCV isolate 350 (0.9446), MCV isolate 339 (0.7976).

\section{Determination of cut-off-values (COVs)}

Net absorbance values were calculated by subtracting the mean absorbance value of the negative control from the mean raw absorbance value read at $450 \mathrm{~nm}$. COVs were determined by ranking net absorbance values and determining the inflection point for each antigen (Figure S1). The GOV for the VP1 antigens of SV40, BKV, JCV, LPV, KIV, and MCV isolate $350 \mathrm{VP1}$ antigens was 0.2 . The $\mathrm{COV}$ for the $\mathrm{VP1}$ antigen of MCV isolate 339 was 0.22 . The COV for WUV VP1 antigen was 0.25.

\section{Recombinant GST-VP1 capsomere-based competition assay}

To determine sera crossreactivity between two or more VP1 proteins, soluble VP1 proteins were purified and plasma samples were pre-adsorbed using these proteins to compete potential crossreactive antibodies in the ELISA assay. Specifically, VP1 capsomere proteins were added to a 1:50 dilution of plasma and incubated for $1 \mathrm{hr}$ at $4^{\circ} \mathrm{C}$ prior to incubating samples with immobilized test antigen. Initially, VP1 capsomeres were titrated to determine the concentration needed to effectively compete crossreactive antibodies (Figure 2). From this titration experiment it was determined that $3.0 \mu \mathrm{g} / \mathrm{ml}$ of competing soluble VP1 was sufficient for the competition assays. Competition assays for SV40 seroprevalence were performed on those samples that exhibited coincident seroreactivity for BKV and/or JCV (Figure 3).

\section{Statistical analysis}

Seroprevalence values were rounded to the nearest integer. Chi square and Fischer's exact tests were performed to determine whether there was a difference in seroprevalence for any of the

\section{References}

1. Gardner SD, Field AM, Coleman DV, Hulme B (1971) New human papovavirus (B.K.) isolated from urine after renal transplantation. Lancet 1: 1253-1257.

2. Padgett BL, Walker DL, ZuRhein GM, Eckroade RJ, Dessel BH (1971) Cultivation of papova-like virus from human brain with progressive multifocal leucoencephalopathy. Lancet 1: 1257-1260.

3. Allander T, Andreasson K, Gupta S, Bjerkner A, Bogdanovic G, et al. (2007) Identification of a third human polyomavirus. J Virol 81: 4130-4136.

4. Gaynor AM, Nissen MD, Whiley DM, Mackay IM, Lambert SB, et al. (2007) Identification of a novel polyomavirus from patients with acute respiratory tract infections. PLoS Pathog 3: e64. doi:10.1371/journal.ppat.0030064.

5. Feng H, Shuda M, Chang Y, Moore PS (2008) Clonal integration of a polyomavirus in human Merkel cell carcinoma. Science 319: 1096-1100.

6. Abedi Kiasari B, Vallely PJ, Corless CE, Al-Hammadi M, Klapper PE (2008) Agerelated pattern of KI and WU polyomavirus infection. J Clin Virol 43: 123-125.

7. Yuan XH, Xu ZQ, Xie ZP, Gao HC, Zhang RF, et al. (2008) [WU polyomavirus and KI polyomavirus detected in specimens from children with acute respiratory tract infection in China]. Zhonghua Shi Yan He Lin Chuang Bing Du Xue Za Zhi 22: 21-23.

8. Bialasiewicz S, Whiley DM, Lambert SB, Wang D, Nissen MD, et al. (2007) A newly reported human polyomavirus, $\mathrm{KI}$ virus, is present in the respiratory tract of Australian children. J Clin Virol 40: 15-18.

9. Payungporn S, Chieochansin T, Thongmee C, Samransamruajkit R, Theamboolers A, et al. (2008) Prevalence and molecular characterization of WU/KI polyomaviruses isolated from pediatric patients with respiratory disease in Thailand. Virus Res 135: 230-236.

10. Neske F, Blessing K, Ullrich F, Prottel A, Wolfgang Kreth H, et al. (2008) WU polyomavirus infection in children, Germany. Emerg Infect Dis 14: 680-681.

11. Abed Y, Wang D, Boivin G (2007) WU polyomavirus in children, Canada. Emerg Infect Dis 13: 1939-1941.

12. Han TH, ChungJY, Koo JW, Kim SW, Hwang ES (2007) WU polyomavirus in children with acute lower respiratory tract infections, South Korea. Emerg Infect Dis 13: $1766-1768$.
VP1 antigens tested with regard to gender. We stratified the pediatric and adult population by age, as shown in Table 1 . Significant differences in cumulative seroprevalence among the age categories were determined by comparing standard errors in each group (Figure 1). SAS version 9.0 was used for all statistical analyses performed.

\section{Supporting Information}

Figure S1 Determination of inflection points for VP1 antigens assayed utilizing the VP1-GST ELISA A) SV40 VP1; B) BKV VP1; C) JCV VP1; D) LPV VP1; E) KIV VP1; F) WUV VP1; G) MCV VP1 isolate 350; H) MCV VP1 isolate 339.

Found at: doi:10.1371/journal.ppat.1000363.s001 (0.43 MB PDF)

Figure S2 Alignment of primary amino acid sequences for the VP1 proteins of MCV isolate 350 and MCV isolate 339. Variable loop regions are highlighted.

Found at: doi:10.1371/journal.ppat.1000363.s002 (0.07 MB PDF)

Table S1 Age distribution of SV40 sero-reactive samples after competition with heterologous VP1 capsomeres.

Found at: doi:10.1371/journal.ppat.1000363.s003 (0.03 MB PDF)

\section{Acknowledgments}

We thank Michael Pawlita for providing plasmids for BKV, JCV, and LPV, David Wang and Tobias Allander for the WUV and KIV VP1 clones, and Denise Galloway for providing the plasmid for MCV strain 339. Additionally, we thank Daniel Ambruso of Bonfil's Blood Center for providing blood samples for the adult population.

\section{Author Contributions}

Conceived and designed the experiments: JMK RLG. Performed the experiments: JMK SR. Analyzed the data: JMK. Contributed reagents/ materials/analysis tools: JMK MW RLG. Wrote the paper: JMK RLG. Aided in editing drafts of the manuscript: SR MW.

13. Lin F, Zheng M, Li H, Zheng C, Li X, et al. (2008) WU polyomavirus in children with acute lower respiratory tract infections, China. J Clin Virol 42: 94-102.

14. Lin F, Zheng MY, Li H, Zheng CH, Rao GF, et al. (2008) [Discovery and identification of WU polyomavirus in children from Zhejing region]. Bing Du Xue Bao 24: 69-71.

15. Sloots TP, Whiley DM, Lambert SB, Nissen MD (2008) Emerging respiratory agents: New viruses for old diseases? J Clin Virol 42: 233-243.

16. zur Hausen H, Gissmann L (1979) Lymphotropic papovaviruses isolated from African green monkey and human cells. Med Microbiol Immunol 167: 137-153.

17. Herrmann M, Oppenlander M, Pawlita M (1995) Fast and High-Affinity Binding of B-Lymphotropic Papovavirus to Human B-Lymphoma Cell Lines. journal of Virology 69: 6797-6804.

18. Pawlita M, Mosthaf L, Clad A, Gruss P (1984) Genome structure and host range restriction of the lymphotropic papovavirus (LPV): identification of a viral lymphocyte specific enhancer element. Curr Top Microbiol Immunol 113: 26-30.

19. Pawlita M, Lenoir G, zur Hausen H (1987) Host range restriction of the lymphotropic papova virus (LPV) in cells of human hematopoietic origin. Haematologica 72: 71.

20. Viscidi RP, Clayman B (2006) Serological cross reactivity between polyomavirus capsids. Adv Exp Med Biol 577: 73-84.

21. Sehr P, Muller M, Hopfl R, Widschwendter A, Pawlita M (2002) HPV antibody detection by ELISA with capsid protein L1 fused to glutathione S-transferase. J Virol Methods 106: 61-70.

22. Sehr P, Zumbach K, Pawlita M (2001) A generic capture ELISA for recombinant proteins fused to glutathione S-transferase: validation for HPV serology. J Immunol Methods 253: 153-162.

23. Knowles WA, Pipkin P, Andrews N, Vyse A, Minor P, et al. (2003) Populationbased study of antibody to the human polyomaviruses BKV and JCV and the simian polyomavirus SV40. J Med Virol 71: 115-123.

24. Knowles WA (2006) Discovery and epidemiology of the human polyomaviruses BK virus (BKV) and JC virus (JCV). Adv Exp Med Biol 577: 19-45. 
25. Jung M, Krech U, Price PC, Pyndiah MN (1975) Evidence of chronic persistent infections with polyomaviruses (BK type) in renal transplant recipients. Arch Virol 47: 39-46.

26. Walker DL, Padgett BL (1983) The epidemiology of human polyomaviruses. Prog Clin Biol Res 105: 99-106.

27. Rziha HJ, Bornkamm GW, zur Hausen H (1978) BK virus: I. Seroepidemiologic studies and serologic response to viral infection. Med Microbiol Immunol (Berl) 165: 73-81.

28. Hogan TF, Borden EC, McBain JA, Padgett BL, Walker DL (1980) Human polyomavirus infections with JC virus and $\mathrm{BK}$ virus in renal transplant patients. Ann Intern Med 92: 373-378.

29. Viscidi RP, Rollison DE, Viscidi E, Clayman B, Rubalcaba E, et al. (2003) Serological cross-reactivities between antibodies to simian virus 40 , BK virus, and JC virus assessed by virus-like-particle-based enzyme immunoassays. Clin Diagn Lab Immunol 10: 278-285.

30. Stolt A, Sasnauskas K, Koskela P, Lehtinen M, Dillner J (2003) Seroepidemiology of the human polyomaviruses. J Gen Virol 84: 1499-1504.

31. Lundstig A, Dillner J (2006) Serological diagnosis of human polyomavirus infection. Adv Exp Med Biol 577: 96-101.

32. Hamilton RS, Gravell M, Major EO (2000) Comparison of antibody titers determined by hemagglutination inhibition and enzyme immunoassay for JC virus and BK virus. J Clin Microbiol 38: 105-109.

33. Ren L, Gonzalez R, Xie Z, Zhang J, Liu C, et al. (2008) WU and KI polyomavirus present in the respiratory tract of children, but not in immunocompetent adults. J Clin Virol 43: 330-333.

34. Bialasiewicz S, Whiley DM, Lambert SB, Jacob K, Bletchly C, et al. (2008) Presence of the newly discovered human polyomaviruses KI and WU in Australian patients with acute respiratory tract infection. J Clin Virol 41: 63-68.

35. Shuda M, Feng H, Kwun HJ, Rosen ST, Gjoerup O, et al. (2008) T antigen mutations are a human tumor-specific signature for Merkel cell polyomavirus. Proc Natl Acad Sci U S A 105: 16272-16277.
36. Garneski KM, Warcola AH, Feng Q, Kiviat NB, Leonard JH, et al. (2008) Merkel Cell Polyomavirus Is More Frequently Present in North American than Australian Merkel Cell Carcinoma Tumors. J Invest Dermatol.

37. Becker JC, Houben R, Ugurel S, Trefzer U, Pfohler C, et al. (2008) MC Polyomavirus Is Frequently Present in Merkel Cell Carcinoma of European Patients. J Invest Dermatol 129: 248-250.

38. Takemoto KK, Segawa K (1983) A new monkey lymphotropic papovavirus: characterization of the virus and evidence of a related virus in humans. Prog Clin Biol Res 105: 87-96.

39. Delbue S, Tremolada S, Branchetti E, Elia F, Gualco E, et al. (2008) First identification and molecular characterization of lymphotropic polyomavirus in peripheral blood from patients with leukoencephalopathies. J Clin Microbiol 46: 2461-2462.

40. Grinnell BW, Martin JD, Padgett BL, Walker DL (1983) Naturally occurring and passage-induced variation in the genome of JC virus. Prog Clin Biol Res 105: 61-77.

41. Brinster RL, Chen HY, Messing A, van Dyke T, Levine AJ, et al. (1984) Transgenic mice harboring SV40 T-antigen genes develop characteristic brain tumors. Cell 37: 367-379.

42. Palmiter RD, Chen HY, Messing A, Brinster RL (1985) SV40 enhancer and large- $T$ antigen are instrumental in development of choroid plexus tumours in transgenic mice. Nature 316: 457-460.

43. Gerber P, Kirschstein RL (1962) SV40-induced ependymomas in newborn hamsters. I. Virus-tumor relationships. Virology 18: 582-588.

44. Kirschstein RL, Gerber P (1962) Ependymomas produced after intracerebral inoculation of SV40 into new-born hamsters. Nature 195: 299-300.

45. Vilchez RA, Kozinetz CA, Arrington AS, Madden CR, Butel JS (2003) Simian virus 40 in human cancers. Am J Med 114: 675-684. 